\title{
Research on Urban Warfare Environment Visibility and Model Building
}

\author{
Yongwang Zhou', 2, a, Lingkui Zhang ${ }^{1, \text { b }}$, Linhai Yao, c, Pengfei Dang ${ }^{1}$ \\ ${ }^{1}$ Xian Surveying and Mapping Institution, Xian Shanxi 710045, China \\ ${ }^{2}$ Information Engineering University, Zhengzhou Henan 450052, China \\ a doctor_zhou@163.com, b myliquor@126.com, c15771779786@163.com
}

Keywords: urban, visibility algorithm, thematic maps, view sphere.

\begin{abstract}
Urban visibility plays an importance role in urban warfare. In MOUT, commanders need taking the urban visibility situation into consideration. In this paper, it analyzes the influences of the urban visibility from aspects of military operation, weapon equipment and war-participators. Based on the usual methods, it studied the LOS visualization algorithm based on RSG elevation data and extended to stereoscopic field calculation. The visual model of urban visualization is established by using three-dimensional sphere-of-view method, put forward the design ideas of city operational visualization thematic map, making examples of observation map city visibility map.
\end{abstract}

\section{Introduction}

The visibility condition in urban environment plays an important role in urban warfare. In the aspect of operation command, urban visibility analysis can be used to find the best position of observation point. Either party which occupied the best sight point will get more awareness on the whole city. In terms of weapons and equipment efficiency, better visibility condition is beneficial to develop indirect firearms superiority. Through the visibility analysis, they can determine the enemy soldiers shooting blind area, find obvious navigation landmarks for cruise vehicle, unmanned robot and other mechanical equipment. In terms of personnel protection, visibility analysis can help finding hidden area for troops; improving the chances of survival in complex urban battlefield environment.

In the field of terrain visibility and vision analysis, Kim and O'sullivan [1,2] had done lots of research on visibility of three-dimensional terrain, making a foundation for understanding of visibility for later generations. Urban visibility analysis is developed on the basis of the terrain analysis. Without considering topographic factors, Batty [3] carried out visibility analysis on the city skyline using 2D method. Chang-lin Yin [4] analyzed the city plane horizon using terrain data. Based on studying variety of methods of spatial visibility calculation, Shen Ying and Li Lin [7] put forward model and indicator for urban form analysis by using visibility analysis. Otherwise, the model and indicators are too complex to calculate. Yang PPJ[5] researched the spatial visibility evaluation method, the key research is located in the point of view of hemisphere horizon body on the ground, established the urban visual effect evaluation indicators, but lack consideration of viewpoints at different heights on visual horizon.

\section{Urban Warfare and Urban Warfare Visibility}

\subsection{Characteristics of Urban Warfare Visibility}

In urban warfare operations, cause of the tall buildings and complex terrain, combatants' observation, command and synergy, motoring actions are difficult, the urban warfare under the information condition of has its own characteristics on battle environment visibility.

Firstly, the urban environment is complex, many factors influencing urban visibility. The biggest influencing factors are the urban constructions. A city is composed of natural and social systems, in which there will be all kinds of high and low different various kinds of building structure, and convenient transportation system and other facilities such as power supply facilities etc. In urban 
environment, all kinds of building facilities configuration, the complexity of layout influence observers' eyesight and affect visibility condition.

Secondly, in urban warfare, because of small contact area for participants, they would be face to face when get in reach. It is very hard to operate combat command, cooperate and coordinate.

Finally, in urban operations the air fire support is essential for both sides. But because of the disorder buildings in the city, whose height changes drastically, combat helicopters that participate in urban warfare need effective use of these building to conceal their actions. While the objects within pilot horizon changes quickly, causing trek, may cause a risk to safety, they requires significant landmarks as a navigation mark in the process of flight.

\subsection{The influence of visibility on urban operations}

Due to complex and various urban terrains, urban environment visibility seriously influences urban operations, weapons using and combatants' security.

(1) Visibility of urban environmental directly affects the urban combat operations in many aspects. In aspect of reconnaissance, the visibility of the urban environment causes some restriction. The purpose of reconnaissance is to find out the visibility condition of urban warfare environment and its impact on the urban combat command and urban warfare implementation; Combat commanders and staff officers conduct operational headquarters location selection, position setting, maneuvering route planning and other activities according to visibility condition of urban warfare environment. The warfighters need to deploy observation post, choose the right enemy close to the ground and hidden places, according to the visibility condition.

(2) In terms of weapons and equipment using, heavy weapons such as tanks in the urban battlefield environment are seriously limited, the warfighter horizon and shooting range are limited in the armored vehicle, increasing risk of attacked by the hidden enemies; Helicopter pilots need to exploit buildings which are beneficial for hidden flying. During the flight, There must be a series of navigation marks in urban battlefield to ensure the correct route.

\section{Three-Dimensional Visibility Indicators of Urban Environment Based on View Sphere}

The visibility conditions in different areas are not same. It is necessary to use a quantitative indicator to describe the visibility in different areas. Using flat horizon algorithm, it can get the visible area within the scope of plane horizon. The visibility of a point can be get by the following formula:

$$
\delta=\frac{S_{v}}{S}
$$

$\delta$ is visibility indicator, $S_{v}$ is the visible area, $S$ is the total area of plane horizon scope.

From above formula, the indicator expressed proportion of around elevation points that can be seen from standing point. But, the formula would be invalid if the around points are partially visible. Therefore, in order to measure visibility in three-dimensional space in urban environment, it established a model of Three-dimensional visibility indicator based on view sphere in this paper.

\subsection{The basic idea and algorithm}

Basic idea of the algorithm is as follows: taking the viewpoint as the central point, it simulates the area into a three dimensional sphere. The scope of sphere is determined by the central point $\mathrm{O}$ and the length of sight line R. The view sphere would be divided in two parts, visible part and invisible part, while some buildings stand in line of sight. Visibility indicator T shows the ratio of visible part of volume to the total volume of view sphere. The process of algorithm shows as below: 


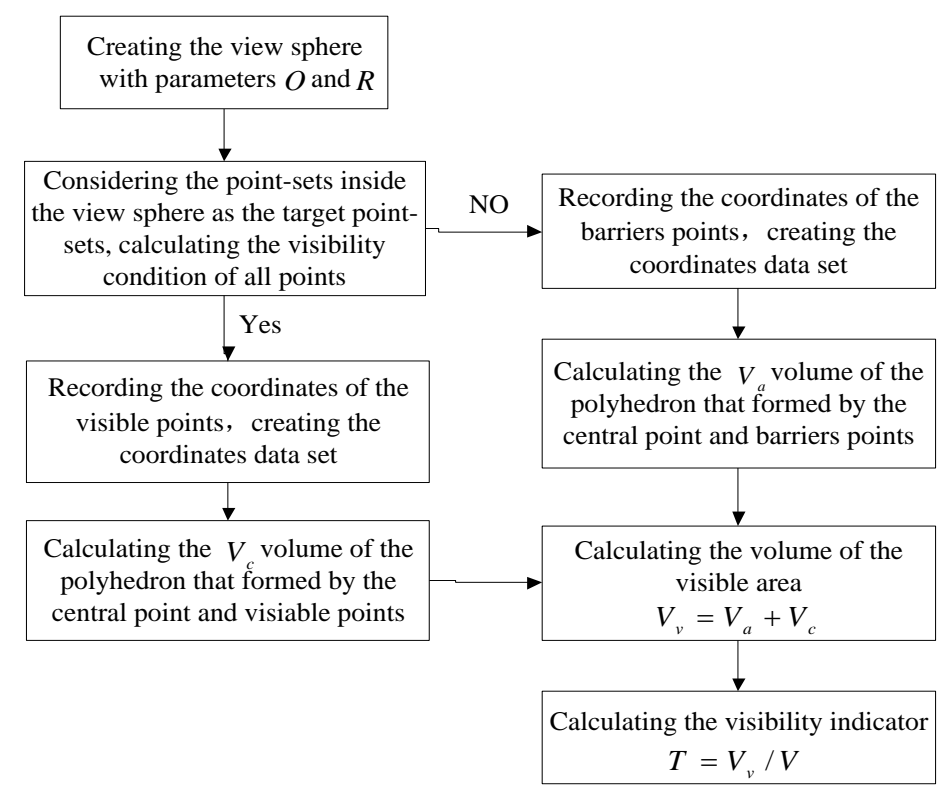

Fig.1 Flow chart of algorithm of 3d visibility indicators

The algorithm is analyzed in detail as fallowing:

(1) It built the view-sphere with observing location as the central point $O\left(X_{O}, Y_{O}, Z_{O}\right)$ and defined sight length as the radius $R$. The space equation of view-sphere defines as below:

$\left(X-X_{O}\right)^{2}+\left(Y-Y_{O}\right)^{2}+\left(Z-Z_{O}\right)^{2}=R^{2}$

(2) Taking point-sets inside the view sphere as the target point-sets, creating a sight line $L_{i}$ (figure 2) from central point $O$ to $S_{i}\left(S_{i}\right.$ is one point of $S$ ), we judge the visibility condition between the two points by comparing the two slopes. If the two points are not visible to each other, we calculate the cross point of $L_{i}$ and $Q_{i}\left(X_{Q i}, Y_{Q i}, Z_{Q i}\right)$ (which is the nearest obstacle elevation point from $O$ ), then calculate the coordinate and elevation of obstacle point $P_{i}$.

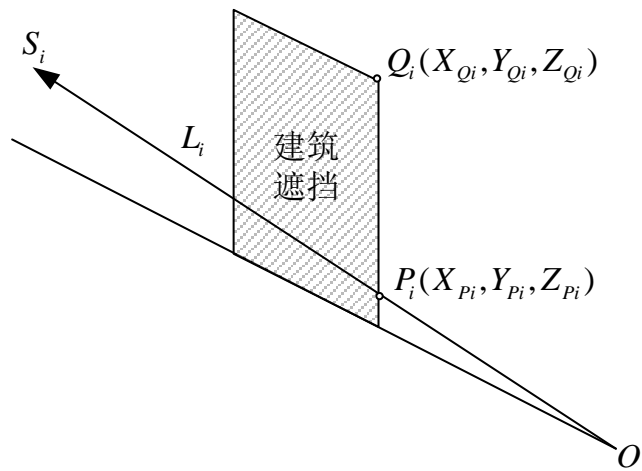

Fig. 2 calculating the cross point of sight line and obstacle point

It uses the following equation to calculate the line determined by the points $O$ and $S_{i}$ :

$$
\frac{\left(X-X_{S i}\right)}{\left(X_{O}-X_{S i}\right)}=\frac{\left(Y-Y_{S i}\right)}{\left(Y_{O}-Y_{S i}\right)}=\frac{\left(Z-Z_{S i}\right)}{\left(Z_{O}-Z_{S i}\right)}
$$

(3) It traverses all the points on the view sphere by changing the horizontal angle and horizontal angle, and then obtains the visual point-sets and obstacle point-sets (figure 3 ). 


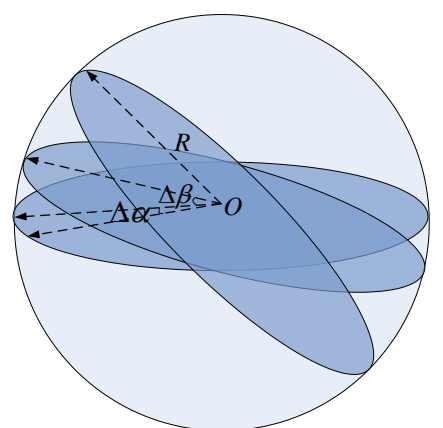

Fig. 3 traversing the all the target points in view sphere

(4) If $O$ and $S_{i}$ are visual to each, it considers the visual area of a cone, its volume $\Delta V_{c}$; if not, the view visual is the tetrahedron established by $O$ and $P_{i}$, its volume $\Delta V_{a}$ (figure 4).

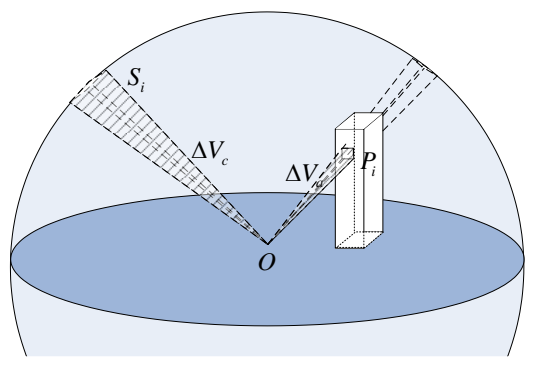

Fig.4 instruction to calculate the volume of visual area

The visibility indicator $T$ is worked out through the following formulas:

$$
\begin{aligned}
& V_{v}=\sum \Delta V_{a}+\sum \Delta V_{c} \\
& T=\frac{V_{v}}{\frac{2}{3} \pi R^{3}}
\end{aligned}
$$

\subsection{Optimization of the algorithm}

In the original algorithm, it needs large amount of calculation, because in the process of calculation it needs to traverse all points. Considering the characteristics of RSG data, it can adjust the horizontal angle by the judgment that weather the elevation point hinders the sight line, not necessary to change the horizontal angle step by step.

As shown in figure $5, L_{i}$ is hindered by the elevation point $\mathrm{A}$, so the line of sight horizontal angle changes $\theta$ directly. $Z_{Q A}$ is elevation of point $\mathrm{A}, d_{i}$ is the distance between $O$ and $\mathrm{A}$.

$$
\theta=\arctan \frac{Z_{Q A}}{d_{i}}
$$
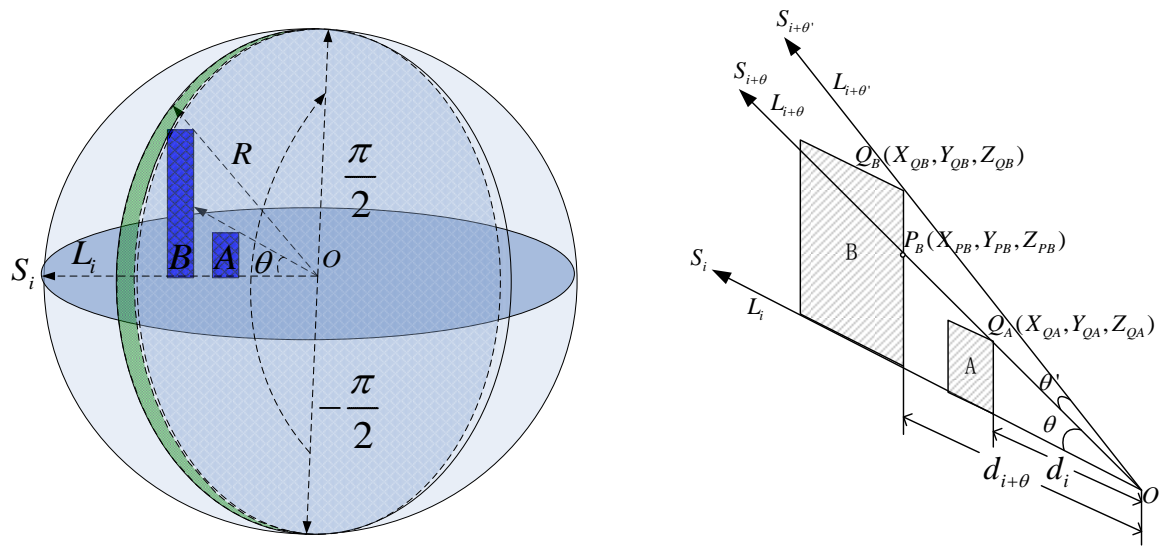

Fig. 5 optimization of the algorithm

Finally, it calculates the volume of the viewing area and visibility indicator $T$ with the same 
method above.

\subsection{Validation of the visibility indicators}

In this paper, It conducted a test to compare the three dimensional visibility indicator $T$ and with flat horizon visibility indicator $\delta$. The buildings in figure 6(a) have usual height, while in figure6 (b) the buildings have doubled height. The calculating results in the following table show that the three dimensional visibility indicator $T$ is more effective.

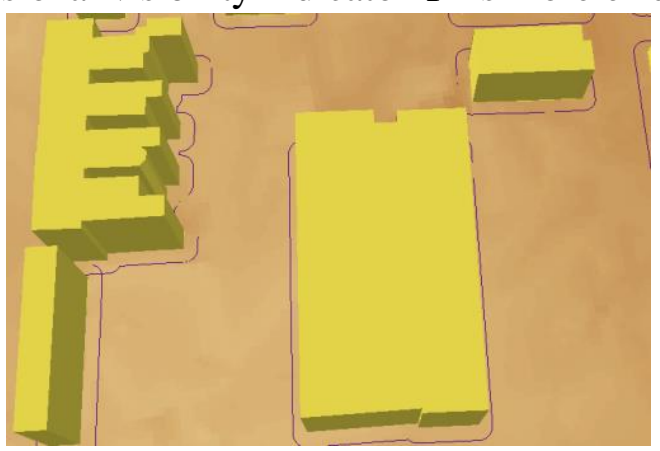

Fig.6 Comparing of the visibility indicator $T$ and $\delta$

Table 1. Calculating result

\begin{tabular}{|c|c|c|c|}
\hline & (a) & (b) & (b)Height*2 \\
\hline$T$ & 0.82 & 0.73 & 0.65 \\
\hline$\delta$ & 0.63 & 0.53 & 0.53 \\
\hline
\end{tabular}

\section{The practice of visual thematic map in urban warfare}

The city visibility algorithm research above can be used in city operations thematic map design, the primary elements of visual thematic map in urban warfare are operational activities, weapons, combat personnel visible degree and range, which is analyzed by using city DEM data and visibility calculation method.

The regional observation of observation post is used as an example in the article to design a visual thematic map for observation post. The high and low buildings in city, always make blind sight to the observation post in three-dimensional region, so to drawing a view map for observation post, stereo analysis must be used as well as point and surface analysis for visual and non-visual range of buildings caused by the change of the height of buildings in the view of three-dimensional city. The design of visual thematic map observation post, one hand will express visibility to the surface region, on the other hand will be different to the stereo view around the key viewpoint level caused by the expression of objects.(As shown in Figure 7)

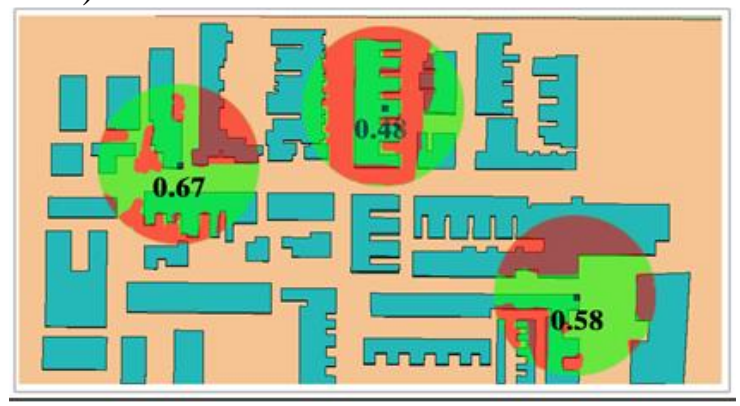

Fig.7(a)The visual area of in observation post on plane 


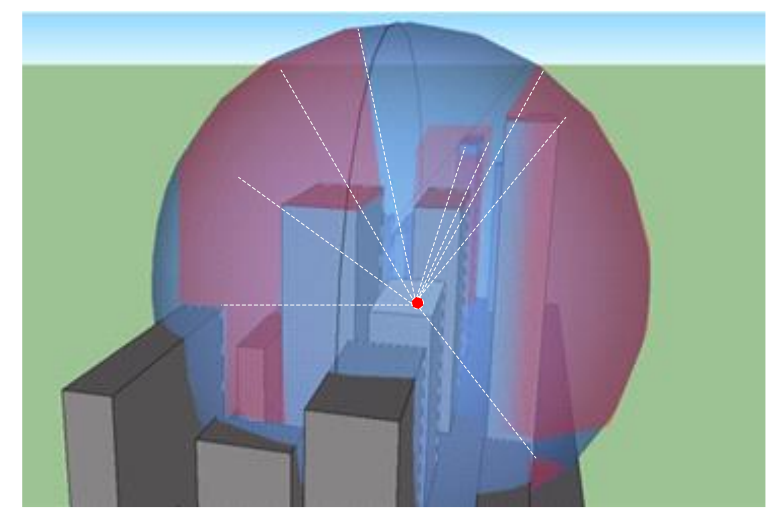

Fig.7(b)The visual area of in observation post on plane

The city visual distribution of thematic map is used as another example. Commanders need to study the situation of the target cities before the war, including the distribution of the visibility of the city. To grasp the visibility of the city, commanders would do well in disposing troops and weapons, conserving manpower to win the war. Defense operations commanders in the city for example, would deploy few squads in the long sight place, but for the short sight, it is necessary to consider more to control the whole area.

To design a city visibility distribution thematic map, points in the map need to be separated into groups, each point must be analyzed by stereo analysis and evaluated by the city visibility indication, and then the result can be used to render the whole map. Regions which have deep color showing good visibility, others are not, this research offers visibility distribution of urban battlefield to commanders, so that they can handle the war and make right decisions.(As shown in Figure 8)

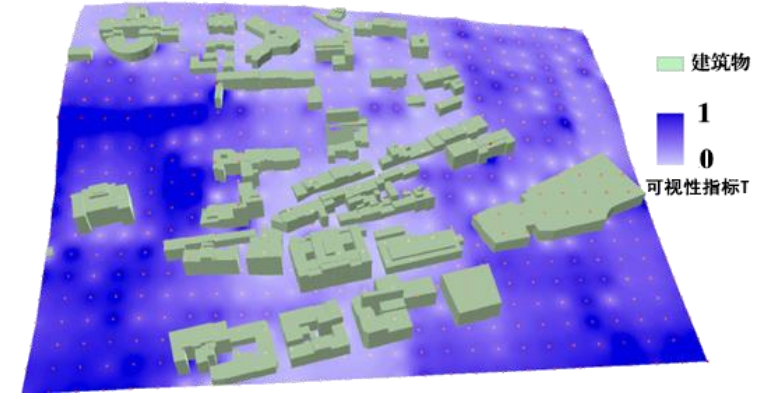

Fig. 8 The visibility condition distribution thematic map in urban enviroment

\section{Conclusion}

In this paper, we study the visibility of urban combat at first, and discuss the impact of visibility on the city operation from three aspects, city operations, weapons and equipment. The visual model of urban visualization is established by using three-dimensional sphere-of-view method, put forward the design ideas of city operational visualization thematic map, making examples of observation map city visibility map.

\section{References}

[1]. Kim Y H, Rana S, Wise S. exploring multiple view-shed analysis using terrain features and optimization techniques [J]. Computers and Geosciences, 2004. NO.30, p. 1019-1032.

[2]. O'sullivan D, Turner A. Visibility graphs and land-scape visibility analysis [J]. Int. J. geographical information science, 2001, NO.15 (3), p. 221-237.

[3]. Batty M. Exploring isovist fields: space and shape in architectural and urban morphology [J]. Environment and Planning B: Planning and Design, 2001. NO.28, p. 123-150.

[4]. Zhou Yongwang, Tian Chaoqian, LI Min. Urban Visibility Analysis Using 3D Space Syntax [J]. Computer Engineering and Applications, 2014. NO.50, p.241-243. 
[5]. Yang PPJ, Putra S Y, Li W. Viewsphere: a GIS-Based 3D visibility analysis for urban design evaluation [J]. Environment and Planning B: Planning and Design, 2007. NO.34, p. 971-992.

[6]. Floriani L D, Magillo P. Algorithms for visibility computation on terrains: a survey [J]. Environment and Planning B: Planning and Design, 2003. NO.30 (5), p.709-728.

[7]. Ying Shen, Li Lin. The research on key technology of spatial visibility analysis and application [J]. Journal of Wuhan University, 2005.

[8]. Wang Huishan, Chen Lin, Yu Weiyu. A Thematic Map Making Mode Based on GIS [J]. Plateau Earthquake Research, 2011. NO.23 (2), p.49-53.

[9]. Wan Wenli, Hu Jiapei, Liu Xuejun. Three-dimensional GIS visibility Analysis Based on Urban DEM [J]. Computer Engineering, 2011. NO.3, p.376-379.

[10]. Leila De Floriani, Paola Magillo. Visibility algorithms on triangulated digital terrain models [J]. International Journal of Geographical Information Science, 1994. NO.8 (1). P.13-41.

[11]. Leila De Floriani, Paola Marzano, Enrico Puppo. Line-of-sight communication on terrain models [J]. International Journal of Geographical Information Science, 1994. NO.8 (4), P.329-342.

[12]. Aronov B, Guibas L, Teichmann M, et al. Visibility queries in simple polygons and applications [J]. Discrete and Computational Geometry, 2002. NO.27, p. 461-483.

[13]. SULEIMAN Wassim, JOLIVEAU Thierry, FAVIER Eric. 3D Urban Visibility Analysis with Vector GIS Data[J]. In GISRUK, University of Portsmouth, UK, 2011.

[14]. Phil Bartie, Femke Reitsma, Simon Kinghamb, Steven Mills. Advancing visibility modelling algorithms for urban environments [J]. Computers environment and urban systems, 2010. 